\title{
First-order reactant of homogeneous dusty fluid turbulence prior to the final period of decay in a rotating system for the case of multi-point and multi-time at four-point correlation
}

\author{
M. Abu Bkar Pk ${ }^{1, ~ *, ~ M . ~ M o n u a r ~ H o s s a i n ~}{ }^{2}$, M. Abul Kalam Azad ${ }^{3}$ \\ ${ }^{1}$ Department of Applied Mathematics, University of Rajshahi, Rajshahi-6205, Bangladesh \\ ${ }^{2}$ Department of Civil \& Environmental Engineering, Uttara University, Dhaka-1230, Bangladesh \\ ${ }^{3}$ Department of Applied Mathematics, University of Rajshahi, Rajshahi-6205, Bangladesh
}

\section{Email address:}

abubakarpk_ru@yahoo.com (M. A. Bkar Pk), milon4215@gmail.com (M. M. Hossain), azad267@gmail.com (M. A. K. Azad)

\section{To cite this article:}

M. Abu Bkar Pk, M. Monuar Hossain, M. Abul Kalam Azad. First-Order Reactant of Homogeneous Dusty Fluid Turbulence Prior to the Final Period of Decay in a Rotating System for the Case of Multi-Point and Multi-Time at Four-Point Correlation. Pure and Applied Mathematics Journal. Vol. 3, No. 4, 2014, pp. 78-86. doi: 10.11648/j.pamj.20140304.11

\begin{abstract}
In this paper following Deissler's approach and taking Fourier transform, the decay for the concentration of a dilute contaminant undergoing a first-order chemical reaction in dusty fluid homogeneous turbulence at times prior to the ultimate phase in a rotating system for the case of multi-point and multi-time at four point correlation is studied. Here two and three point correlations between fluctuating quantities have been considered and the quadruple correlations are ignored in comparison to the second and third order correlations. Taking Fourier transform the correlation equations are converted to spectral form. Finally, integrating the energy spectrum over all wave numbers we obtained the decay law for the concentration fluctuations of first order reactant in homogeneous dusty fluid turbulence prior to the final period of decay in a rotating system for the case of multi-point and multi-time at four-point correlation.
\end{abstract}

Keywords: Correlation Function, Deissler's Method, Dusty Fluid Turbulence, First Order Chemical Reactant, Fourier-Transformation, Multi-point and multi-time, Navier-Stokes Equation, Rotating System

\section{Introduction}

The introduction of the Chemical reactions occur in the gas phase, in solution in a variety of solvents, at gas-solid and other interfaces, in the liquid state, and in the solid state. It is sometimes convenient to work with amounts of substances instead of with concentrations. The essential characteristic of turbulent flows is that turbulent fluctuations are random in nature. Chemical reaction as used in chemistry, chemical engineering, physics, fluid mechanics, heat and mass transport. In recent year; the motion of dusty viscous fluids in a rotating system has developed rapidly. The motion of dusty fluid occurs in the movement of dust -laden air, in problems of fluidization, in the use of dust in a gas cooling system and in the sedimentation problem of tidal rivers. The behavior of dust particles in a turbulent flow depends on the concentrations of the particles and the size of the particles with respect to the scale of turbulent fluid. In geophysical flows, the system is usually rotation with a constant velocity, such large Scale flows are generally turbulent. The Coriolis Effect is caused by the rotation of the Earth and the inertia of the mass experiencing the effect. The most commonly encountered rotating reference frame in the Earth. Because the Earth completes only one rotation per day, this force causes moving objects on the surface of the Earth to appear to change direction to the right in the northern hemisphere, and to the left in the southern and it has great significance in Astrophysics, Stellar dynamics, Earth sciences, Meteorology, Physical geology and Oceanography. Deissler $(1958,1960)$ developed a theory 'on the decay of homogeneous turbulence before the final period'. Loeffler and Deissler (1961) studied the temperature fluctuations in homogeneous turbulence before the final period. Kishore and Golsefid (1988) obtained and expression for the effect of 
Coriolis force on acceleration covariance in MHD turbulent flow of a dusty incompressible fluid. Kumar and Patel (1974) studied the first order reactants in homogeneous turbulence before the final period of decay. Kumar and Patel (1975) extended their problem (1974) on first-order reactants in homogeneous turbulence for the case of multi-point and multi-time concentration correlation. In (1991), Sarker and Kishore studied the decay of MHD turbulence at times before the final period using Chandrasekhar's (1951) invariant theory of isotropic turbulence in magneto-hydrodynamics relation. Shimomura and Yoshizawa (1986) derived expressions for statistical analysis of an isotropic turbulent viscosity in a rotating system. Shimomura (1989) also studied a statistical equation model of turbulent scalar flux in a rotating system. Sarker and Ahmed (2011) pointed out that the fiber motion in dusty fluid turbulent flow with two point correlation. Corrsin (1951) obtained on the spectrum of isotropic temperature fluctuations in isotropic turbulence. Azad et al. (2011) also studied the statistical theory of certain distribution functions in MHD turbulent flow for velocity and concentration undergoing a first order reaction in a rotating system. M.A.Bkar Pk et al. (2012) derived the decay of energy of MHD turbulence for four-point correlation. M.A.Bkar Pk et al. (2013) also studied First-order reactant in homogeneou turbulence prior to the ultimate phase of decay for four-point correlation in presence of dust particle. M.Monuar Hossain et al.(2014) obtained the homogeneous fluid turbulence before the final period of decay for four-point correlation in a rotating system for first order reactant. Bkar $\mathrm{Pk}$ et al., (2013) explained that the decay of MHD turbulence prior to the ultimate phase in presence of dust particle for four-point correlation. Bkar Pk et al., (2013) also studied that the decay of dusty fluid MHD turbulence for four-point correlation in a rotating system.

M.Monuar Hossain et al.(2014) obtained their work at four-point single time. But in our work, First-order reactant of homogeneous dusty fluid turbulence prior to the final period of decay in a rotating system for the case of multi-point and multi-time at four-point correlation has been studied.

Here, we have considered two-point, three-point and four-point correlation equations and solved these equations after neglecting fifth-order correlation terms. Finally we obtained the decay law of energy fluctuations of concentration of dilute contaminant undergoing a first order chemical reaction in dusty fluid homogeneous turbulence in a rotating system for the case of multi-point and multi-time at four-point correlation comes out to the form

$$
\left\langle X^{2}\right\rangle=\exp \left(-2 \mathrm{RT}_{\mathrm{m}}\right)\left\{A T_{m}^{-3 / 2}+\exp \left[-\left(2 \varepsilon_{m k i} \Omega_{m}-f Q\right)\right] B T_{m}^{-5}\right\} .
$$

where $\left\langle X^{2}\right\rangle$ denotes the concentration fluctuation energy. It is seen that the demolition of the impurity is more rapid than that in the case of pure mixing. This result has been shown in the figure also.

\section{Material and Methods}

Following Kumar and Patel (1974) for first-order chemical reaction in homogeneous dusty fluid turbulence in a rotating system could be written as:

$$
\begin{gathered}
\frac{\partial u_{i}}{\partial t}+u_{k} \frac{\partial u_{i}}{\partial x_{k}}=-\frac{1}{\rho} \frac{\partial p}{\partial x_{k}}+v \frac{\partial^{2} u_{i}}{\partial x_{k} \partial x_{k}}-R u_{i} \\
-2 \varepsilon_{m k i} \Omega_{m} u_{i}+f\left(u_{i}-v_{i}\right)
\end{gathered}
$$

The subscripts can take on the values 1, 2, and 3 .

Here,

$u_{i}(\hat{x})=$ a random function of position and time at a point $\mathrm{p}$,

$u_{k}(\hat{x}, t)=$ turbulent velocity,

$R=$ constant reaction rate,

$D=$ diffusivity,

$t=$ time,

$\mathcal{E}_{m k i}=$ alternating tensor,

$\Omega_{m}=$ constant angular velocity components,

$f=\frac{k N}{\rho}$, dimension of frequency,

$N=$ constant number density of dust particle,

$m_{s}=\frac{4}{3} \pi R_{s}{ }^{3} \rho_{s}$, mass of single spherical dust particle of radius $R_{s}$,

$\rho_{s}=$ constant density of the material in dust particle,

$v=$ Kinematics viscosity,

$\rho=$ Fluid density,

$p(\hat{x}, t)=$ Pressure fluctuation,

$u_{k}=$ turbulent velocity component,

$v_{i}=$ dust particle velocity component,

$x_{k}=$ space-coordinate and repeated subscript in a term indicates a summation of terms, with the subscripts successively taking on the values 1,2 , and 3 .

\section{Correlations and Spectral Equations}

Under the limitations that

(i) For multi-point and multi-time the turbulence and the concentration fields are homogeneous.

(ii) For multi-point and multi-time the chemical reaction and the local mass transfer have no effect on the velocity field and

(iii) The reaction rate and the diffusivity are constant for multi-point and multi-time.

Differential equation governing the concentration of a dilute contaminant undergoing a first-order chemical reaction we take the Navier-Stokes equations at the point $P$ and the concentration equation at $\mathrm{P}^{\prime}$ and separated by the vector $\hat{r}$ could be written as 


$$
\begin{gathered}
\frac{\partial X}{\partial t}+u_{k} \frac{\partial X}{\partial x_{k}}=D \frac{\partial^{2} X}{\partial x_{k} \partial x_{k}}-R X \\
\frac{\partial X^{\prime}}{\partial t^{\prime}}+u_{k}^{\prime} \frac{\partial X^{\prime}}{\partial x_{k}^{\prime}}=D \frac{\partial^{2} X^{\prime}}{\partial x_{k}^{\prime} \partial x_{k}^{\prime}}-R X^{\prime}
\end{gathered}
$$

where $X(\hat{x}, t)$ is a random function of position and time. The other symbols are as usual. Multiplying equation (2) by $X^{\prime}$, equation (3) by $X$, and averaging, we get

$$
\begin{gathered}
\frac{\partial\left\langle X X^{\prime}\right\rangle}{\partial t}+\frac{\partial\left\langle u_{k} X X^{\prime}\right\rangle}{\partial x_{k}}=D \frac{\partial^{2}\left\langle X X^{\prime}\right\rangle}{\partial x_{k} \partial x_{k}}-R\left\langle X X^{\prime}\right\rangle \\
\frac{\partial\left\langle X X^{\prime}\right\rangle}{\partial t^{\prime}}+\frac{\partial\left\langle u_{k}^{\prime} X X^{\prime}\right\rangle}{\partial x_{k}^{\prime}}=D \frac{\partial^{2}\left\langle X X^{\prime}\right\rangle}{\partial x_{k}^{\prime} \partial x_{k}^{\prime}}-R\left\langle X X^{\prime}\right\rangle
\end{gathered}
$$

where the conditions of continuity and the fact that the quantities at a point at a particular time are independent of the positions at the other points have been utilized. For multi-point and multi-time using the transformations.

$$
\frac{\partial}{\partial x_{k}}=-\frac{\partial}{\partial r_{k}}, \frac{\partial}{\partial x_{k}^{\prime}}=\frac{\partial}{\partial r_{k}}, \frac{\partial}{\partial t}=\left(\frac{\partial}{\partial t}\right)_{\Delta t}-\frac{\partial}{\partial \Delta t}, \frac{\partial}{\partial t^{\prime}}=\frac{\partial}{\partial \Delta t},
$$

in to equation (4) and (5), we obtain

$$
\begin{gathered}
\frac{\partial\left\langle X X^{\prime}\right\rangle}{\partial t}+\frac{\partial\left\langle u_{k} X X^{\prime}\right\rangle}{\partial r_{k}}(-\hat{r},-\Delta t, t+\Delta t)-\frac{\partial\left\langle u_{k} X X^{\prime}\right\rangle}{\partial r_{k}}(\hat{r}, \Delta t, t)=2 D \frac{\partial^{2}\left\langle X X^{\prime}\right\rangle}{\partial r_{k} \partial r_{k}}-2 R\left\langle X X^{\prime}\right\rangle \\
\frac{\partial\left\langle X X^{\prime}\right\rangle}{\partial \Delta t}+\frac{\partial\left\langle u_{k} X X^{\prime}\right\rangle}{\partial r_{k}}(-\hat{r},-\Delta t, t+\Delta t)=D \frac{\partial^{2}\left\langle X X^{\prime}\right\rangle}{\partial r_{k} \partial r_{k}}-R\left\langle X X^{\prime}\right\rangle
\end{gathered}
$$

In order to reduce equations (6) and (7) to spectral form by using three-dimensional Fourier transform

$$
\begin{array}{r}
\left\langle X X^{\prime}(\hat{r}, \Delta t, t)\right\rangle=\int_{-\infty}^{\infty} \theta^{\prime}(\hat{k}, \Delta t, t) \exp (i \hat{k} . \hat{r}) d \hat{k} \\
\left\langle X X^{\prime}(\hat{r}, \Delta t, t)\right\rangle=\int_{-\infty}^{\infty} \phi_{k}(\hat{k}, \Delta t, t) \exp (i \hat{k} . \hat{r}) d \hat{k}
\end{array}
$$

We get

$$
\begin{gathered}
\frac{\partial \theta^{\prime}}{\partial t}+\left(2 D k^{2}+2 R\right) \theta^{\prime}=i k_{k} \phi_{k}(\hat{k}, \Delta t, t)+i\left(-k_{k}\right) \phi_{k}\left(-\hat{k}_{k},-\Delta t, t+\Delta t\right) \\
\frac{\partial \theta^{\prime}}{\partial \Delta t}+\left(D k^{2}+R\right) \theta^{\prime}=-i k_{k} \phi_{k}(-\hat{k},-\Delta t, t+\Delta t)
\end{gathered}
$$

where $\theta^{\prime}(\hat{k}, \Delta t, t)$ is a function of the three independent variables $\hat{k}, \Delta t, t$; that is obtained by initial conditions.

For the ultimate phase of homogeneous turbulence decompose, the third-order correlations can be ignored in comparison to the second-order correlations. With this approximation, the solutions of equations (10) and (11) may be obtained as

$$
\begin{aligned}
& \theta^{\prime}=f_{1}(\hat{k}, \Delta t) \exp \left[-\left(2 D k^{2}+2 R\right)\left(t-t_{0}\right)\right] \\
& \left.\theta^{\prime}=f_{2}(\hat{k}, t) \exp \left[-\left(D k^{2}+R\right) \Delta t\right)\right]
\end{aligned}
$$

For consistent solution of equations (12) and (13) we must have

$$
G(k)=f(k) \exp \left[\left(-2 D k^{2}+2 R\right)\left(t-t_{0}+\frac{\Delta t}{2}\right)\right]
$$

where $G(k)=2 \pi k^{2} \theta^{\prime}$ is the concentration spectrum function. We evaluate $f(k)$ by Corrsin (1951) i.e. $f(k)=N_{0} k^{2} / \pi$, where a constant $N_{0}$ is depending on initial condition. Thus, we obtain

$$
G(k)=\frac{N_{0} k^{2}}{\pi} \exp \left[\left(-2 D k^{2}+2 R\right)\left(t-t_{0}+\frac{\Delta t}{2}\right)\right]
$$

By integrating equation (15) with respect to $k$, we obtain

$$
\left\langle X X^{\prime}\right\rangle\left(\hat{r}, t_{m}\right)=\frac{N_{0} D^{1 / 2}}{4 \sqrt{4 \pi}\left(t_{m}-t_{0}\right)^{3 / 2}} \exp \left[-\left\{\frac{2 c\left(t_{m}-t_{0}\right)+r^{2}}{8 D\left(t_{m}-t_{0}\right)}\right\}\right]
$$

where $t_{m}=t+\Delta t / 2$.

\section{Three-Point, Three-Time Correlations and Equations}

Under the same assumptions as before, we take the Navier-Stokes equation for dusty homogeneous fluid turbulence in a rotating system at the point $P$ and the concentration equations at $P^{\prime}$ and $P^{\prime \prime}$, following Kumar and Patel (1975) as

$$
\begin{gathered}
\frac{\partial u_{i}}{\partial t}+u_{k} \frac{\partial u_{i}}{\partial x_{k}}=-\frac{1}{\rho} \frac{\partial p}{\partial x_{k}}+v \frac{\partial^{2} u_{i}}{\partial x_{k} \partial x_{k}}-R u_{i} \\
-2 \varepsilon_{m k i} \Omega_{m} u_{i}+f\left(u_{i}-v_{i}\right) \\
\frac{\partial X^{\prime}}{\partial t^{\prime}}+u_{k}^{\prime} \frac{\partial X^{\prime}}{\partial x_{k}^{\prime}}=D \frac{\partial^{2} X^{\prime}}{\partial x_{k}^{\prime} \partial x_{k}^{\prime}}-R X^{\prime} \\
\frac{\partial X^{\prime \prime}}{\partial t^{\prime \prime}}+u_{k}^{\prime \prime} \frac{\partial X^{\prime \prime}}{\partial x_{k}^{\prime \prime}}=D \frac{\partial^{2} X^{\prime \prime}}{\partial x_{k}^{\prime} \partial x_{k}^{\prime \prime}}-R X^{\prime \prime}
\end{gathered}
$$

Multiplying equation (17) by $X X^{\prime \prime},(18)$ by $u_{i} X^{\prime \prime}$ and (19) by $u_{i} X^{\prime}$ and then taking space averages, we obtain.

$$
\frac{\partial}{\partial t}\left\langle u_{i} X^{\prime \prime} X^{\prime \prime \prime}\right\rangle+\frac{\partial}{\partial x_{k}}\left\langle u_{i} u_{k} X^{\prime} X^{\prime \prime}\right\rangle=-\frac{1}{\rho} \frac{\partial}{\partial x_{k}}\left\langle p X^{\prime} X^{\prime \prime}\right\rangle+v \frac{\partial^{2}}{\partial x_{k} \partial x_{k}}\left\langle u_{i} X^{\prime} X^{\prime \prime}\right\rangle-R\left\langle u_{i} X^{\prime} X^{\prime \prime}\right\rangle-2 \varepsilon_{m k i} \Omega_{m}\left\langle u_{i} X^{\prime} X^{\prime \prime}\right\rangle+f\left[\left\langle u_{i} X^{\prime} X^{\prime \prime}\right\rangle-\left\langle v_{i} X^{\prime} X^{\prime \prime}\right\rangle\right]
$$




$$
\begin{aligned}
\frac{\partial}{\partial t^{\prime}}\left\langle u_{i} X^{\prime} X^{\prime \prime}\right\rangle+\frac{\partial}{\partial x_{k}^{\prime}}\left\langle u_{i} u_{k}^{\prime} X^{\prime} X^{\prime \prime}\right\rangle & =D \frac{\partial^{2}}{\partial x_{k}^{\prime} \partial x_{k}^{\prime}}\left\langle u_{i} X^{\prime} X^{\prime \prime}\right\rangle-R\left\langle\mathrm{u}_{\mathrm{i}} X^{\prime} X^{\prime \prime}\right\rangle \\
\frac{\partial}{\partial t^{\prime \prime}}\left\langle u_{i} X^{\prime} X^{\prime \prime}\right\rangle+\frac{\partial}{\partial x_{k}^{\prime \prime}}\left\langle u_{i} u_{k}^{\prime \prime} X^{\prime} X^{\prime \prime}\right\rangle & =D \frac{\partial^{2}}{\partial x_{k}^{\prime \prime} \partial x_{k}^{\prime \prime}}\left\langle u_{i} X^{\prime} X^{\prime \prime}\right\rangle-R\left\langle\mathrm{u}_{\mathrm{i}} X^{\prime} X^{\prime \prime}\right\rangle
\end{aligned}
$$

For three-point and three-time, using the transformations

$$
\frac{\partial}{\partial x_{k}}=-\left(\frac{\partial}{\partial r_{k}}+\frac{\partial}{\partial r_{k}^{\prime}}\right), \frac{\partial}{\partial x_{k}^{\prime}}=\frac{\partial}{\partial r_{k}}, \frac{\partial}{\partial x_{k}^{\prime \prime}}=\frac{\partial}{\partial r_{k}^{\prime}}, \frac{\partial}{\partial t} t^{\prime}, t^{\prime \prime}=\left(\frac{\partial}{\partial t}\right)_{\Delta t, \Delta t^{\prime}}-\frac{\partial}{\partial \Delta t}-\frac{\partial}{\partial \Delta t^{\prime}}, \frac{\partial}{\partial t^{\prime}}=\frac{\partial}{\partial \Delta t}, \frac{\partial}{\partial t^{\prime \prime}}=\frac{\partial}{\partial \Delta t^{\prime}}
$$

Into equation (20)-(22), we get

$$
\begin{gathered}
\frac{\partial}{\partial t}\left\langle u_{i} X^{\prime} X^{\prime \prime}\right\rangle-\left(\frac{\partial}{\partial r_{k}}+\frac{\partial}{\partial r_{k}^{\prime}}\right)\left\langle u_{i} u_{k} X^{\prime} X^{\prime \prime}\right\rangle+\frac{\partial}{\partial r_{k}}\left\langle u_{i} u_{k}^{\prime} X^{\prime} X^{\prime \prime}\right\rangle+\frac{\partial}{\partial r_{k}^{\prime}}\left\langle u_{i} u_{k}^{\prime \prime} X^{\prime} X^{\prime \prime}\right\rangle \\
=-\frac{1}{\rho}\left(\frac{\partial}{\partial r_{k}}+\frac{\partial}{\partial r_{k}^{\prime}}\right)\left\langle p X^{\prime} X^{\prime \prime}\right\rangle+v\left(\frac{\partial}{\partial r_{k}}+\frac{\partial}{\partial r_{k}^{\prime}}\right)^{2}\left\langle u_{i} X^{\prime} X^{\prime \prime}\right\rangle+D\left(\frac{\partial^{2}}{\partial r_{k} \partial r_{k}}+\frac{\partial^{2}}{\partial r_{k}^{\prime} \partial r_{k}^{\prime}}\right)\left\langle u_{i} X^{\prime} X^{\prime \prime}\right\rangle \\
-\left(2 R+2 \varepsilon_{m k i} \Omega_{m}\right)\left\langle u_{i} X^{\prime} X^{\prime \prime}\right\rangle+f\left[\left\langle u_{i} X^{\prime} X^{\prime \prime}\right\rangle-\left\langle v_{i} X^{\prime} X^{\prime \prime}\right\rangle\right] \\
\frac{\partial}{\partial \Delta t}\left\langle u_{i} X^{\prime} X^{\prime \prime}\right\rangle+\frac{\partial}{\partial r_{k}}\left\langle u_{i} u_{k}^{\prime} X^{\prime} X^{\prime \prime}\right\rangle=D \frac{\partial^{2}}{\partial r_{k}^{\prime} \partial r_{k}^{\prime}}\left\langle u_{i} X^{\prime} X^{\prime \prime}\right\rangle-R\left\langle X^{\prime} u_{i} X^{\prime \prime}\right\rangle \\
\frac{\partial}{\partial \Delta t^{\prime}}\left\langle u_{i} X^{\prime} X^{\prime \prime}\right\rangle+\frac{\partial}{\partial r_{k}^{\prime}}\left\langle u_{i} u_{k}^{\prime \prime} X^{\prime} X^{\prime \prime}\right\rangle=D \frac{\partial^{2}}{\partial r_{k}^{\prime \prime} \partial r_{k}^{\prime \prime}}\left\langle u_{i} X^{\prime} X^{\prime \prime}\right\rangle-R\left\langle X^{\prime} u_{i} X^{\prime \prime}\right\rangle
\end{gathered}
$$

For four-point and four-time correlations using the six-dimensional Fourier transform of the type

$$
\left\langle X X^{\prime} u_{k}^{\prime \prime}\left(\hat{r}, \hat{r}^{\prime}, \Delta t, \Delta t^{\prime}, t\right)\right\rangle=\int_{-\infty}^{\infty} \cdot \int_{-\infty}^{\infty} \psi_{k}\left(\hat{k}, \hat{k}^{\prime}, \Delta t, \Delta t^{\prime}, t\right) \exp \left(\hat{k} \cdot \hat{r}+\hat{k}^{\prime}, \hat{r}^{\prime}\right) d \hat{k} d \hat{k}^{\prime}
$$

and with the fact that,

$$
\left\langle X^{\prime} X^{\prime \prime} v_{k}\right\rangle=\int_{-\infty}^{\infty} \cdot \int_{-\infty}^{\infty} \psi_{k}^{\prime}\left(\hat{k}, \hat{k}^{\prime}, \Delta t, \Delta t^{\prime}, t\right) \exp \left(\hat{k} \cdot \hat{r}+\hat{k}^{\prime} \cdot \hat{r}^{\prime}\right) d \hat{k} d \hat{k}^{\prime}
$$

and the assumption that the quintuple correlations representing the transfer terms in equations (23) - (25) can be neglected as they decay faster than the lower-order correlation terms. Then the equation (23) - (25) in Fourier space can be written as

$$
\begin{gathered}
\frac{\partial \psi_{i}}{\partial t}\left(\hat{k}, \hat{k}^{\prime}, \Delta t, \Delta t^{\prime}, t\right)+D\left[\left(1+N_{S}\right) k^{2}+2 N_{s} k k^{\prime} \cos \left\{\left(1+N_{s}\right) k^{\prime 2} \quad \begin{array}{l}
\text { correlations are also neglected. Integrating equations } \\
(26)-(28) \text { between } t_{o} \text { and } t, \text { we obtain }
\end{array}\right.\right. \\
\left.\left.+\left(2 R+2 \varepsilon_{m k i} \Omega_{m}-f Q\right) / D\right\}\right] \psi_{i}\left(\hat{k}, \hat{k}^{\prime}, \Delta t, \Delta t^{\prime}, t\right)=0 \\
\psi_{i}=f_{i} \exp \left\{-D\left[\left(1+N_{s}\right) k^{2}+2 N_{s} k k^{\prime} \cos \theta+\left(1+N_{s}\right) k^{\prime 2}+\left(2 R+2 \varepsilon_{m k i} \Omega_{m}-f Q\right) / D\right]\left(t-t_{0}\right)\right\} \\
\psi_{i}=g_{i} \exp \left\{\left[-D\left(k^{2}+R / D\right)\right] \Delta t\right\} \\
\psi_{i}=h_{i} \exp \left\{\left[-D\left(k^{\prime 2}+R / D\right)\right] \Delta t^{\prime}\right\} .
\end{gathered}
$$

where $N_{s}=v / D$, the Schmidt number and

$$
\left\langle X^{\prime} X^{\prime \prime} v_{l}\right\rangle=L \psi_{i}^{\prime}\left\langle X^{\prime} X^{\prime \prime} v_{l}\right\rangle, 1-L=Q
$$

As the pressure force terms are related to higher-order correlations, therefore, these along with the quadruple

For these relations to be consistent, we have

$$
k_{i} \psi_{i}=k_{i}\left(\psi_{i}\right)_{0} \exp \left[-D\left\{\left(1+N_{s}\right)\left(k^{2}+k^{\prime 2}\right)\left(t-t_{0}\right)+k^{2} \Delta t+k^{\prime 2} \Delta t^{\prime}+2 N_{s} k k^{\prime} \cos \theta\left(t-t_{0}\right)\right.\right.
$$




$$
\left.\left.+(2 R / D)\left[t-t_{0}+\left(\Delta t+\Delta t^{\prime}\right) / 2\right]+\left[\left(2 \varepsilon_{m k i} \Omega_{m}-f Q\right) / D\right]\left(t-t_{0}\right)\right\}\right]
$$

where the subscript 0 refers to the value of $\psi_{i}$ at $t=t_{o}, \Delta \mathrm{t}=$ $\Delta \mathrm{t}^{\prime}=0$ and $\theta$ is the angle between $k$ and $k^{\prime}$.The relation between $\phi_{i}$ and $\psi_{i}$ is given by

$$
k_{i} \phi_{i}(\hat{k}, \Delta t, t)=\int_{-\infty}^{\infty} k_{i} \psi_{i}\left(\hat{k}, \Delta t, \hat{k}^{\prime}, 0, t\right) d k^{\prime}
$$

Substituting equations (30) and (29) into equation (10),

$$
\begin{gathered}
W=\int_{0}^{\infty} i k_{i}\left(\psi_{i}\right)_{0} k^{2} k^{\prime 2}(2 \pi)^{2} \exp \left\{-D\left[\left(1+N_{S}\right) k^{2}\left(t-t_{o}+\frac{\Delta t}{t+N_{S}}\right)+k^{\prime 2}\left(1+N_{S}\right)\left(t-t_{o}\right)+\left[\left(2 \varepsilon_{m k i} \Omega_{m}-f Q\right) / D\right]\left(t-t_{0}\right)+2 R / D+\right.\right. \\
\left.\left.(2 R / D)\left(t-t_{o}+\frac{\Delta t}{2}\right)\right\}\right]\left\{\int_{-1}^{1} \exp \left[-2 N_{s} D k k^{\prime}\left(t-t_{0}\right) \cos \theta\right] d k^{\prime}+\int_{0}^{\infty}\left[i\left(-k_{i}\right) \psi_{i}\left(-\hat{k},-\hat{k}^{\prime}\right)\right]_{0}(2 \pi)^{2} k^{2} k^{\prime 2} \exp \left\{-D\left[\left(1+N_{s}\right) k^{2}\left(t-t_{o}+\frac{\Delta t}{1+N_{S}}\right)\right.\right.\right. \\
\left.\left.-k^{\prime 2}\left(1+N_{s}\right)\left(t-t_{0}+\Delta t\right)+\left[\left(2 \varepsilon_{m k i} \Omega_{m}-f Q\right) / D\right]\left(t-t_{0}\right)+\frac{2 R}{D}\left(t-t_{0}+\frac{\Delta t}{2}\right)\right]\right\} \times \int_{-1}^{1} \exp \left[-2 N_{s} D k k^{\prime}\left(t-t_{0}+\Delta t\right) \cos \theta\right](d \cos \theta) d k^{\prime}
\end{gathered}
$$

where $d k^{\prime}$ is written as $2 \pi k^{\prime 2} d(\cos \theta) d k^{\prime}$ and the quantity $\left(\psi_{i}\right)_{0}$ depends on the initial conditions of the Substituting equation (33) in (32) and completing the integration, we get turbulence. Now, following Deissler $(1958,1960)$, we take $(2 \pi)^{2} i\left[k_{i} \psi_{i}\left(\hat{k}, \hat{k}^{\prime}\right)_{0}=-\frac{1}{2} \delta_{0}\left(k^{2} k^{\prime 4}-k^{4} k^{\prime 2}\right)\right.$ and

$$
\begin{aligned}
(2 \pi)^{2} i\left[-k_{i} \psi_{i}\left(-\hat{k},-\hat{k}^{\prime}\right)_{0}=\right. & \frac{1}{2} \delta_{0}\left(k^{2} k^{\prime 4}-k^{4} k^{\prime 2}\right) \\
W= & -\frac{\delta_{o} N_{s} \pi^{1 / 2}}{4 D^{3 / 2}\left(t-t_{o}\right)^{3 / 2}\left(1+N_{s}\right)^{5 / 2}} \exp \left[-k^{2} D \frac{1+2 N_{s}}{1+N_{s}}\left(t-t_{o}+\frac{1+N_{s}}{1+2 N_{s}} \Delta t\right)\right. \\
+ & \left.\left(2 \varepsilon_{m k i} \Omega_{m}-f Q\right)\left(t-t_{0}\right)-2 R\left(t-t_{o}+\frac{\Delta t}{2}\right)\right]\left\{\frac{15 k^{4}}{4 N_{s}^{2}\left(t-t_{o}\right)^{2} D^{2}} \frac{N_{s}}{1+N_{s}}+\left[5\left(\frac{N_{s}}{1+N_{s}}\right)^{2}-\frac{3}{2}\right] \times\right. \\
& \left.\frac{k^{6}}{N_{s} D\left(t-t_{o}\right)}+\left[\left(\frac{N_{s}}{1+N_{s}}\right)^{3}-\frac{N_{s}}{1+N_{s}}\right] k^{8}\right\}-\frac{\delta o N_{s} \sqrt{\pi}}{4 D^{3} / 2\left(t-t_{o}+\Delta t\right)^{3 / 2}\left(t+N_{s}\right)^{5 / 2}} \times \\
& \exp \left[-k^{2} D \frac{1+2 N_{s}}{1+N_{s}}\left(t-t_{o}+\frac{N_{s}}{+1+N_{s}} \Delta t\right)+\left(2 \varepsilon_{m k i} \Omega_{m}-f Q\right)\left(t-t_{0}\right)-2 R\left(t-t_{o}-\frac{\Delta t}{2}\right)\right] \\
\times & \left\{\frac{15 k^{4}}{4 D^{2} N_{s}^{2}\left(t-t_{o}+\Delta t\right)^{2}}\left(\frac{N_{s}}{1+N_{s}}\right)+\left[5\left(\frac{N_{s}}{1+N_{s}}\right) 2-\frac{3}{2}\right] \frac{k^{6}}{N_{s} D\left(t-t_{o}+\Delta t\right)}+\left[\left(\frac{N_{s}}{1+N_{s}}\right)^{3}-\frac{N_{s}}{1+N_{s}}\right] k^{8}\right\}
\end{aligned}
$$

This represents the transfer function arising due to the consideration of concentration at three- point and three- time. When $\Delta t=0$ and $R=0$, the expression for reduces to the case of pure mixing. It may also be noted that (for $\Delta t=0$ )

$$
\int W d k=0
$$

Here $W$ satisfies the condition of continuity and homogeneity. Since $\mathrm{W}$ is the measure of transfer of energy and total energy transferred to all wave numbers is to be zero. With the help of equation (31) and (34), we get 


$$
\begin{aligned}
G= & \frac{N_{0} k^{2}}{\pi} \exp \left\{-2\left(k^{2} D+2 R\right)\left(t-t_{o}+\frac{\Delta t}{2}\right)\right\}+\frac{\sqrt{\pi} N_{s}}{D^{3 / 2}\left(1+N_{s}\right)^{7 / 2}} \times \frac{\delta_{0}}{4} \exp \left[-D \frac{1+2 N_{s}}{1+N_{s}} k^{2}\left(t-t_{o}+\frac{\left(1+N_{s}\right) \Delta t}{1+2 N_{s}}\right)+\left(2 \varepsilon_{m k i} \Omega_{m}-f Q\right)\left(t-t_{0}\right)-2 R\left(t-t_{o}+\frac{\Delta t}{2}\right)\right] \\
& \times\left[\frac{3 k^{4}}{2 N_{s} D^{2}\left(t-t_{o}\right)^{5 / 2}}+\frac{\left(7 N_{s}-6\right) k^{6}}{3 D\left(1+N_{s}\right)\left(t-t_{o}\right)^{3 / 2}}-\frac{4\left(3 N_{s}^{2}-2 N_{s}+3\right) k^{8}}{3\left(1+N_{s}\right)^{2}\left(t-t_{o}\right)^{1 / 2}}+\frac{8 D^{1 / 2}\left(3 N_{s}^{2}-2 N_{s}+3\right) k^{9}}{3\left(1+N_{s}\right)^{5 / 2}} F\left(k \sqrt{\frac{\left(t-t_{o}\right) D}{1+N_{s}}}\right)\right]+\frac{\sqrt{\pi} N_{s}}{D^{3 / 2}\left(1+N_{s}\right)^{7 / 2}} \frac{\delta_{o}}{4} \\
& \exp \left[-D\left(\frac{1+2 N_{s}}{1+N_{s}}\right) k^{2}\left(t-t_{o}+\frac{N_{s}}{1+2 N_{s}} \Delta t\right)+\left(2 \varepsilon_{m k i} \Omega_{m}-f Q\right)\left(t-t_{0}\right)-2 R\left(t-t_{o}+\frac{\Delta t}{2}\right)\right] \\
& \times\left[\frac{3 k^{4}}{2 D^{2} N_{s}\left(t-t_{o}+\Delta t\right)^{5 / 2}}+\frac{\left(7 N_{s}-6\right) k^{6}}{3 D\left(1+N_{s}\right)\left(t-t_{o}+\Delta t\right)^{3 / 2}}-\frac{4}{3} \frac{\left(3 N_{s}^{2}-2 N_{s}+3\right) k^{8}}{\left(1+N_{s}\right)^{2}\left(t-t_{o}+\Delta t\right)^{1 / 2}}+\frac{8 D^{1 / 2}\left(3 N_{s}^{2}-2 N_{s}+3\right) k^{9}}{\left(1+N_{s}\right)^{5 / 2}} F\left(k \sqrt{\frac{\left(t-t_{o}+\Delta t\right) D}{1+N_{s}}}\right)\right]
\end{aligned}
$$

where,

$$
\begin{gathered}
F(\omega)=\exp \left(-\omega^{2}\right) \int_{0}^{\omega} \exp \left(x^{2}\right) d x, \\
\omega=k \sqrt{\frac{\left(t-t_{o}\right) D}{1+N_{s}}} \text { or } \mathrm{k}=\sqrt{\frac{\left(\mathrm{t}-\mathrm{t}_{\mathrm{o}}+\Delta t\right) \mathrm{D}}{1+N_{s}}}
\end{gathered}
$$

As in the previous section, by integrating equation (36) with respect to $k$, we obtain

$$
\begin{aligned}
& \left\langle\frac{X X^{\prime}}{2}\left(\Delta t, t_{m}\right)\right\rangle=\int_{0}^{\infty} G d k=\frac{N_{o}}{8 D^{3 / 2} \sqrt{2 \pi}\left(T+\frac{\Delta t}{2}\right)^{3 / 2}} \exp \left[-2 R\left(T+\frac{\Delta t}{2}\right)\right]+\frac{\pi}{D^{6}\left(1+N_{s}\right)\left(1+N_{s}\right)^{5 / 2}} \exp \left[-\left(2 \varepsilon_{m k i} \Omega_{m}-f Q\right)\right] \exp \left[-2 R\left(T+\frac{\Delta t}{2}\right)\right] \\
& \times \frac{\delta_{0}}{4} \frac{9}{16 T^{5 / 2}\left(T+\frac{1+N_{s}}{1+2 N_{s}} \Delta T\right)^{5 / 2}}+\frac{9}{16(T+\Delta T)^{5 / 2}\left(T+\frac{1+N_{s}}{1+2 N_{s}} \Delta T\right)^{5 / 2}}+\frac{5 N_{s}\left(7 N_{s}-6\right)}{16\left(1+2 N_{s}\right) T^{3 / 2}\left(T+\frac{1+N_{s}}{1+2 N_{s}} \Delta T\right)^{7 / 2}} \\
& +\frac{5 N_{s}\left(7 N_{s}-6\right)}{16\left(1+2 N_{s}\right)(T+\Delta T)^{3 / 2}\left(T+\frac{N_{s}}{1+2 N_{s}} \Delta T\right)^{7 / 2}}+\frac{35 N_{s}\left(3 N_{s}^{2}-2 N_{s}+3\right)}{8\left(1+2 N_{s}\right) T^{1 / 2}\left(T+\frac{1+N_{s}}{1+2 N_{s}} \Delta T\right)^{9 / 2}}+\frac{35 N_{s}\left(3 N_{s}^{2}-2 N_{s}+3\right)}{8\left(1+2 N_{s}\right)(T+\Delta T)^{1 / 2}\left(T+\frac{N_{s}}{1+2 N_{s}} \Delta T\right)^{9 / 2}}+ \\
& +\frac{8 N_{s}\left(3 N_{s}^{2}-2 N_{s}+3\right)\left(1+2 N_{s}\right)^{5 / 2}}{3.2^{23 / 2}\left(1+N_{s}\right)^{11 / 2}} \sum_{n=0}^{\infty} \frac{1.3 .5 !(2 n+1) 2^{2 n}\left(1+N_{s}\right)^{s}}{\left(T+\frac{\Delta T}{2}\right)^{(2 n+11) / 2}}+\frac{(\mathrm{T}+\Delta \mathrm{T})^{(2 \mathrm{n}+1) / 2}}{\left(T+\frac{\Delta T}{2}\right)^{(2 n+1) / 2}}
\end{aligned}
$$

where, $T=\left(t-t_{0}\right)$,

For $T_{m}=T+\Delta T / 2$, equation (37) becomes

$$
\begin{gathered}
\left\langle\frac{X X^{\prime}}{2}\left(t_{m}\right)\right\rangle=\exp \left(-2 R T_{m}\right) \frac{N_{0}}{8 \sqrt{2 \pi} D^{3 / 2} T_{m}^{3 / 2}}+\frac{\delta_{0} \pi}{4 D^{6}\left(1+N_{s}\right)\left(1+2 N_{s}\right)} \exp \left[-\left(2 \varepsilon_{m k i} \Omega_{m}-f Q\right)\right] \frac{9}{16} \frac{1}{\left(T_{m}-\frac{\Delta T}{2}\right)^{5 / 2}\left(T_{m}+\frac{\Delta T}{1+2 N_{s}}\right)^{5 / 2}} \\
+\frac{9}{16\left(T_{m}+\frac{\Delta T}{2}\right)^{5 / 2}\left(T_{m}-\frac{\Delta T}{2}\right)^{5 / 2}}+\frac{5 N_{s}\left(7 N_{s}-6\right)}{16\left(1+2 N_{s}\right)} \frac{1}{\left(T_{m}+\frac{\Delta T}{2}\right)^{7 / 2}\left(T_{m}-\frac{\Delta T}{2}\right)^{3 / 2}}+\frac{5 N_{s}\left(7 N_{s}-6\right)}{16\left(1-2 N_{s}\right)} \frac{1}{\left(T_{m}+\frac{\Delta T}{2}\right)^{3 / 2}\left(T_{m}-\frac{\Delta T}{1+2 N_{S}}\right)^{7 / 2}+\ldots}
\end{gathered}
$$

If $\Delta t=0$, equation (38) reduces to the form

$$
\left\langle\frac{X^{2}}{2}\right\rangle=\exp \left(-2 R T_{m}\right)\left\{\frac{N_{o}}{8 \sqrt{2 \pi} D^{3 / 2}} \frac{1}{\mathrm{~T}_{\mathrm{m}}^{3 / 2}}+\frac{\delta_{o} \pi}{2 D^{6}\left(1+N_{s}\right)\left(1+2 N_{s}\right)} \exp \left[-\left(2 \varepsilon_{m k i} \Omega_{m}-f Q\right)\right]\left[\frac{9}{16 T_{m}^{5}}+\frac{5}{16} \frac{N_{s}\left(7 N_{s}-6\right)}{\left(1+2 N_{s}\right) T_{m}^{5}}\right]\right\}
$$

Therefore, 


$$
\left\langle X^{2}\right\rangle=\exp \left(-2 R T_{m}\right)\left[\frac{N_{o}}{4 \sqrt{2 \pi} D^{3 / 2}} \frac{3}{T_{m}^{3 / 2}}+\exp \left[-\left(2 \varepsilon_{m k i} \Omega_{m}-f Q\right)\right] \frac{\delta_{o} \alpha}{D^{6}} \frac{1}{T_{m}^{5}}\right]
$$

where,

$$
\alpha=\frac{\pi}{\left(1+N_{s}\right)\left(1+2 N_{s}\right)}\left[\frac{9}{16}+\frac{5}{16} \frac{N_{s}\left(7 N_{s}-6\right)}{\left(1+2 N_{s}\right)}+\ldots\right] .
$$

Thus, the decay law for the concentration energy fluctuation of homogeneous dusty fluid in a rotating system for multi-point and multi-time prior to the final period may be written as

$$
\left\langle X^{2}\right\rangle=\exp \left(-2 \mathrm{RT}_{\mathrm{m}}\right)\left\{A T_{m}^{-3 / 2}+\exp [-(F-H)] B T_{m}^{-5}\right\}
$$

where

$$
A=\frac{3 N_{0}}{4 \sqrt{2 \pi} D^{3 / 2}}, B=\frac{\delta_{0} \alpha}{D^{6}}, F=2 \varepsilon_{m k i} \Omega_{m} \text { and } H=f Q .
$$

\section{Results and Discussion}

It is noted that $y 1, y 2, y 3, y 4, y 5, y 6$ and $y 7$ are energy decay curves of equation (40). But in the absence of dust particles and non-rotating system $y 1, y 2, y 3, y 4, y 5, y 6, y 7$ are represented by equation (41). Figures 1, 2, and 3 represents energy decay curves of equation (40) and equation (41) for figure 4 . In equation (40) we obtained the concentration fluctuation energy of dusty fluid homogeneous turbulence in a rotating system for multi-point and multi-time. In the absence of corriolis force i.e. if the frame is non-rotating and dust particles are absence then equation (40) becomes

$$
\left\langle X^{2}\right\rangle=\exp \left(-2 \mathrm{RT}_{\mathrm{m}}\right)\left\{A T_{m}^{-3 / 2}+B T_{m}^{-5}\right\}
$$

which was obtained earlier by Kumar and Patel (1974).For large times, the last term of equation (41) becomes negligible and the decay law for the ultimate period becomes $\exp \left(-2 \mathrm{RT}_{\mathrm{m}}\right)\left(A T_{m}^{-3 / 2}\right)$ which in the case of pure-mixing is similar to the law obtained by Corrsin(1953). In Fig.1, we observe that the variation of chemical reaction $(R=3.5,1.75,0.875,0.44,0.22,0.11$ and 0$)$ causes significant changes in the concentration fluctuation decay of energy of dusty fluid homogeneous turbulence in presence of corriolis force for multi-point and multi-time due to $F=2.75$ and $H=1.5$. It is mentioned that the energy decay of the fluid particles decreases with the increases of the chemical reaction $R$ and is maximum at zero. If the system is non-rotating i.e. the corriolis force is absence the energy decay of the fluid particles more rapidly than rotating with dust particle system which indicated in the Fig.2, .In Fig.3, for multi-point and multi-time we observe that in the absence of corriolis force and dust particles energy decay more slowly than with the present of dust particles in a rotating system.

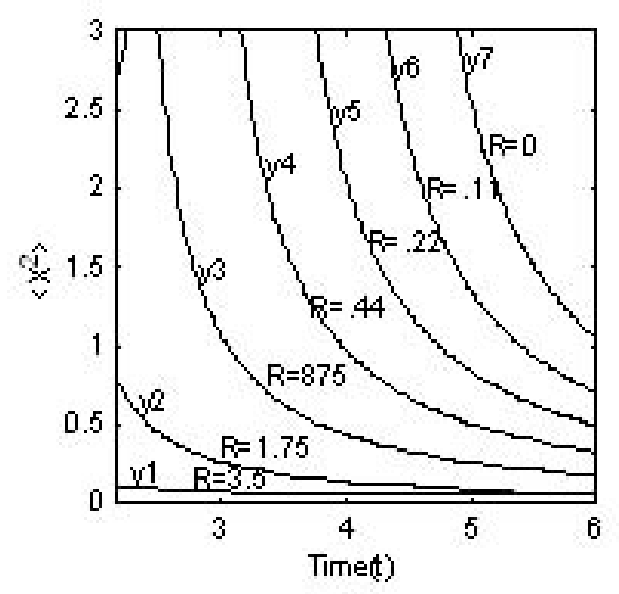

Fig. 1. Energy decay curves for $F=2.75, H=1.5$.

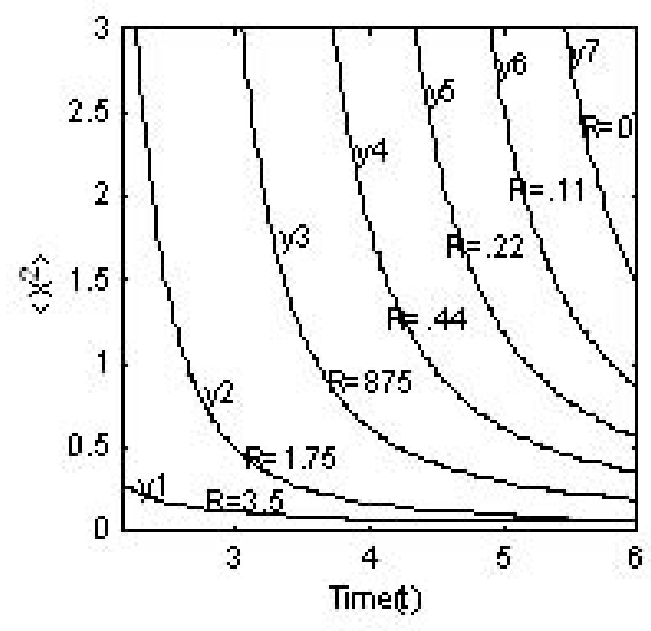

Fig. 2. Energy decay curves for $F=0, H=1.5$.

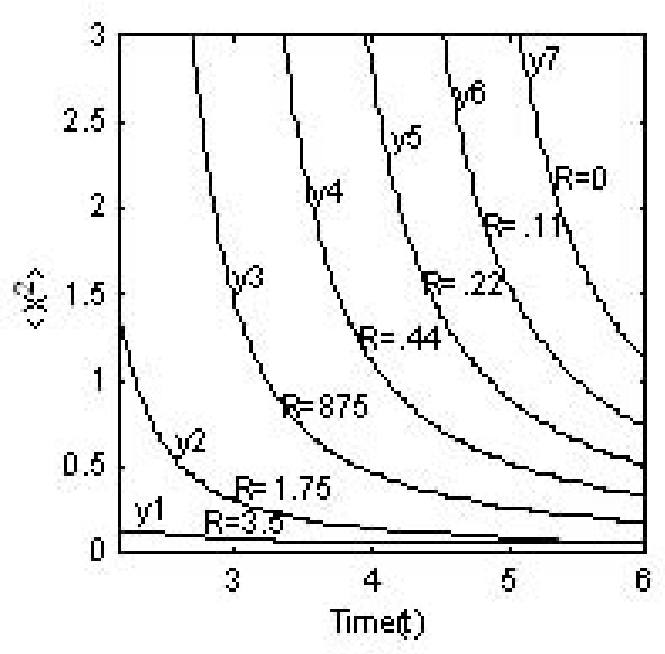

Fig. 3. Energy decay curves for $F=2.75, H=0$. 


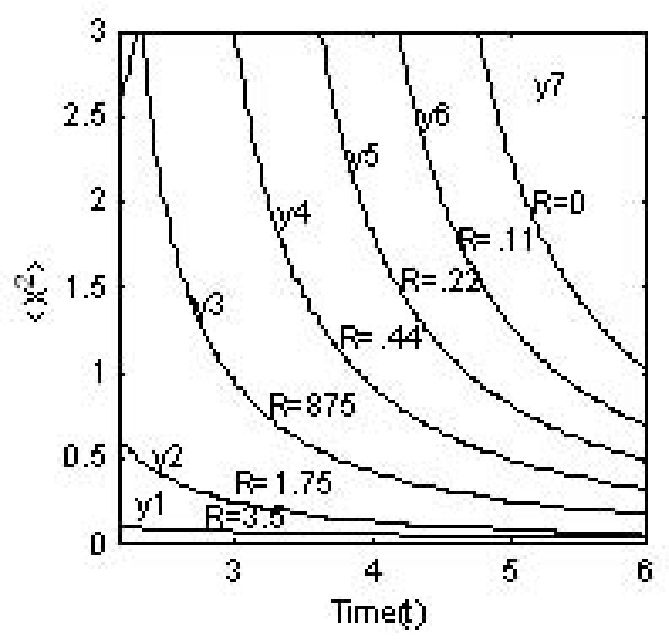

Fig. 4. Energy decay curves for $F=0, H=0$.

In Fig.4, we notice that in the absence of dust particles the energy decay of fluid particles very slowly due to the variation of first-order chemical reaction in homogeneous fluid turbulence for the case of multi-point and multi-time.

\section{Conclusion}

The decomposition of the concentration fluctuation in homogeneous turbulence in the rotating system is more slowly due to absence of dust particles than any other type of chemical reactant. In the case of non rotating system for four point correlation, this study shows that if the concentration selected is the chemical reactant of the first order, then the effect is that the decomposition of the concentration fluctuation in homogeneous turbulence in the presence of dust particle is much more rapid if the system is non-rotating and the faster rate of decomposition is governed by $\exp \left(-2 \mathrm{RT}_{\mathrm{m}}\right)$. In a normal way, it takes a lot of time to get rid of a pollutant in the fluid. The only effective factor in the case of chemical reactant is $\exp \left(-2 \mathrm{RT}_{\mathrm{m}}\right)$ which can be taken as the correcting law for the pure mixing case and may be applied to the data for the case of reactant and thus, the numerical work required for this study has been avoided. From the above figures and discussion, we conclude that for both rotating and non-rotating frame the decomposition of the concentration fluctuation in homogeneous turbulence are increases due to the decreases of the first order chemical reaction in either the presence of dust particles or not and maximum at the point where the chemical reaction is zero.

\section{Acknowledgements}

Authors (M.A.Bkar Pk \&M.A.K.Azad) are grateful to the Department of Applied Mathematics, University of Rajshahi, Bangladesh and author M.M.Hossain is greatfull to the Department of Civil \& Environmental Engineering, Uttara University-Dhaka Bangladesh for giving all facilities and support to carry out this work. The authors are also thankful to the editor and reviewer for their valuable comments and suggestions.

\section{References}

[1] Azad, M.A.K., M.A.Aziz and M. S. Alam Sarker, "The statistical theory of certain distribution functions in MHD turbulent flow for velocity and concentration undergoing a first order reaction in a rotating system.” Bangladesh J. Sci. Ind. Res. 46(1): 59. 2011.

[2] Bkar Pk, M.A., M.S.Alam Sarker and M.A.K. Azad, "Decay of MHD turbulence prior to the ultimate phase in presence of dust particle for four-point correlation." Int .J. of Appl.Math and Mech.9 (10):34-57.2013.

[3] Bkar Pk, M.A., M.A.K. Azad and M.S.Alam Sarker, "Decay of energy of MHD turbulence for four-point correlation." International Journal of Engineering Research \& Technology.1 (9):pp1-13. 2012.

[4] Bkar Pk. M.A., M.A.K.Azad and M.S.Alam.Sarker, "First-order reactant in homogeneous turbulence prior to the ultimate phase of decay for four-point correlation in presence of dust particle,"Res.J.Appl.Sci.Eng. Technol.,5(2):585-595. 2013(a).

[5] Bkar Pk. M.A., M.A.K.Azad and M.S.Alam.Sarker, "Decay of dusty fluid MHD turbulence for four-point correlation in a rotating system.'J.Sci. Res.5 (1):77-90.2013(b).

[6] Chandrasekhar, S., "The invariant theory of isotropic turbulence in magneto-hydrodynamics," Proc. Roy.Soc., London, and A204:435-449. 1951.

[7] Corrsin, S., "On the spectrum of isotropic temperature fluctuations in an isotropic turbulence." J.Apll.Phys 22:469-473.1951.

[8] Deissler, R.G., "On the decay of homogeneous turbulence before the final period." Phys.Fluid, 1:111-121. 1958.

[9] Deissler, R.G., "A theory of decaying homogeneous turbu lence.” Phys. Fluid, 3:176-187. 1960.

[10] Kishore, N, and Y.T. Golsefid. "The effect of Coriolis force on acceleration covariance in MHD turbulent flow of a dusty incompressible fluid." Astr. Space Sci.: 150, 89. 1988.

[11] Kumar, P. and S.R.Patel, "First-order reactant in homogeneous turbulence before the final period of decay." Phys .Fluids, 17: 1362-1368. 1974.

[12] Kumar, P. and S.R.Patel, "On first-order reactant in homogeneous turbulence.”Int. J. Eng. Sci., 13:305-315. 1975.

[13] Loeffler, A.L and R.G. Deissler, "Decay of temperature fluctuations in homogeneous turbulence before the final period.” Int. J. Heat Mass Trans., 1(4): 312-324. 1961.

[14] Hossain.M.M, M.A.Bkar Pk and M.S. A. Sarker "Homogeneous fluid turbulence before the final period of decay for four-point correlation in a rotating system for first-order reactant." American Journal of Theoretical and Applied Statistics, 3(4):81-89. 2014.

[15] Shimomura. Y. and A.Yoshizawa, "Statistical analysis of an isotropic turbulent viscosity in a rotating system.” J. Physical Soc., Japan, 55(6): 1904-1917. 1986.

[16] Shimomura, Y. "A statistical derived two-equation model of turbulent scalar flux in a rotating system.” J .Physical Soc., Japan, 58: 352. 1989. 
[17] Sarker, M. S. A. and N. Kishore, "Decay of MHD turbulence before the final period.” Int. J. Engng Sci., 29(11):1479-1485. 1991.
[18] Sarker. M. S.A, and S.F. Ahmed, 2011. Fiber motion in dusty fluid turbulent flow with two point correlation. J. Sci. Res. 3(2): 283-290. 